\title{
Capsaicin Regulates Voltage-Dependent Sodium Channels by Altering Lipid Bilayer Elasticity
}

\author{
J. A. Lundbæk, P. Birn, S. E. Tape, G. E. S. Toombes, R. Søgaard, Roger E. Koeppe II, \\ S. M. Gruner, A. J. Hansen, and O. S. Andersen \\ Novo Nordisk A/S, Måløv, Denmark (J.A.L., P.B., A.J.H.); Department of Physiology and Biophysics, Weill Medical College \\ (J.A.L., S.E.T., O.S.A.) and Department of Physics (G.E.S.T., S.M.G.), Cornell University, Ithaca, New York; Institute of \\ Biological Psychiatry, St. Hans Hospital, Roskilde, Denmark (J.A.L., R.S.); August Krogh Institute, University of Copenhagen, \\ Denmark (J.A.L.); and Department of Chemistry and Biochemistry, University of Arkansas, Fayetteville, Arkansas (R.E.K.)
}

Received April 13, 2005; accepted June 20, 2005

\begin{abstract}
At submicromolar concentrations, capsaicin specifically activates the TRPV1 receptor involved in nociception. At micro- to millimolar concentrations, commonly used in clinical and in vitro studies, capsaicin also modulates the function of a large number of seemingly unrelated membrane proteins, many of which are similarly modulated by the capsaicin antagonist capsazepine. The mechanism(s) underlying this widespread regulation of protein function are not understood. We investigated whether capsaicin could regulate membrane protein function by changing the elasticity of the host lipid bilayer. This was done by studying capsaicin's effects on lipid bilayer stiffness, measured using gramicidin A ( $\mathrm{gA}$ ) channels as molecular forcetransducers, and on voltage-dependent sodium channels (VDSC) known to be regulated by bilayer elasticity. Capsaicin and capsazepine (10-100 $\mu \mathrm{M})$ increase gA channel appearance rate and lifetime without measurably altering bilayer thick-
\end{abstract}

ness or channel conductance, meaning that the changes in bilayer elasticity are sufficient to alter the conformation of an embedded protein. Capsaicin and capsazepine promote VDSC inactivation, similar to other amphiphiles that decrease bilayer stiffness, producing use-dependent current inhibition. For capsaicin, the quantitative relation between the decrease in bilayer stiffness and the hyperpolarizing shift in inactivation conforms to that previously found for other amphiphiles. Capsaicin's effects on gA channels and VDSC are similar to those of Triton $\mathrm{X}-100$, although these amphiphiles promote opposite lipid monolayer curvature. We conclude that capsaicin can regulate VDSC function by altering bilayer elasticity. This mechanism may underlie the promiscuous regulation of membrane protein function by capsaicin and capsazepine-and by amphiphilic drugs generally.
Capsaicin [ $N$-(4-hydroxy-3-methoxybenzyl)-8-methylnon6 -eneamide] is an important tool in pain research. At submicromolar concentrations, capsaicin specifically activates the TRPV1 receptor involved in pain sensation (Caterina et al., 1997). At micro- to millimolar concentrations, commonly used in pain research, capsaicin modulates a plethora of seemingly unrelated membrane proteins, many of which are similarly affected by the capsaicin antagonist capsazepine ( $N$-[2-(4-chlorophenyl)ethyl]-1,3,4,5-tetrahydro-7,8-dihydroxy-2-H-2-benzazepine-2-carbothioamide; see Table 1).

This work was supported by grants from The Danish Heart Association (to J.A.L.), National Institutes of Health grants GM21342 (to O.S.A.) and RR15569 (to REK), and United States Department of Energy grant DE-FG0297ER62443 (to S.M.G.)

Article, publication date, and citation information can be found at http://molpharm.aspetjournals.org.

doi:10.1124/mol.105.013573.
The mechanism(s) underlying this promiscuous regulation of membrane protein function are not understood, but capsaicin changes the physical properties of lipid bilayers (Meddings et al., 1991; Aranda et al., 1995). The hydrophobic coupling between a membrane protein and the lipid bilayer means that protein conformational changes involving the protein/ bilayer interface (Lee, 2003) perturb the surrounding bilayer with an associated energetic cost (Mouritsen and Bloom, 1984). Because lipid bilayers are elastic bodies (Evans and Hochmuth, 1978), protein function can be regulated by changes in the bilayer elastic properties (Gruner, 1991; Andersen et al., 1992; Lundbæk and Andersen, 1994). To investigate whether the widespread effects of capsaicin could involve changes in bilayer elasticity, we examined its effects on two channel types known to be regulated by the bilayer elastic properties.

ABBREVIATIONS: gA, gramicidin; VDSC, voltage-dependent sodium channel; GA, [Val'1] ${ }^{1}$ gramicidin A (formyl-L-Val-Gly-L-Ala-D-Leu-L-Ala-D-ValL-Val-D-Val-L-Trp-D-Leu-L-Trp-D-Leu-L-Trp-D-Leu-L-Trp-ethanolamine); DPhPC, diphytanoylphosphatidylcholine; DOPC, dioleoylphosphatidylcholine; MOPS, 3-(N-morpholino)propanesulfonic acid; HEK, human embryonic kidney. 
Pharmacologically induced changes in bilayer elasticity can be measured using gramicidin (gA) channels as molecular force transducers. gA channels are formed by trans-bilayer association of two monomers (Fig. 1). When the bilayer's hydrophobic thickness is larger than the channel's hydrophobic length, the bilayer adjust locally to match the channel length. This bilayer deformation imposes a disjoining force on the channel, which varies with bilayer thickness, monolayer spontaneous curvature, and bilayer elastic moduli (Huang, 1986). From an operational standpoint, we define changes in bilayer properties that, at a constant thickness, alter the disjoining force as changes in bilayer stiffness. A decrease in stiffness will decrease this force and increase gA channel appearance rate and lifetime (Lundbæk and Andersen, 1994; Lundbæk et al., 1996; Hwang et al., 2003; Lundbæk et al., 2004). Conversely, an increase in gA channel appearance rate and lifetime allows one to infer that stiffness

\section{A}
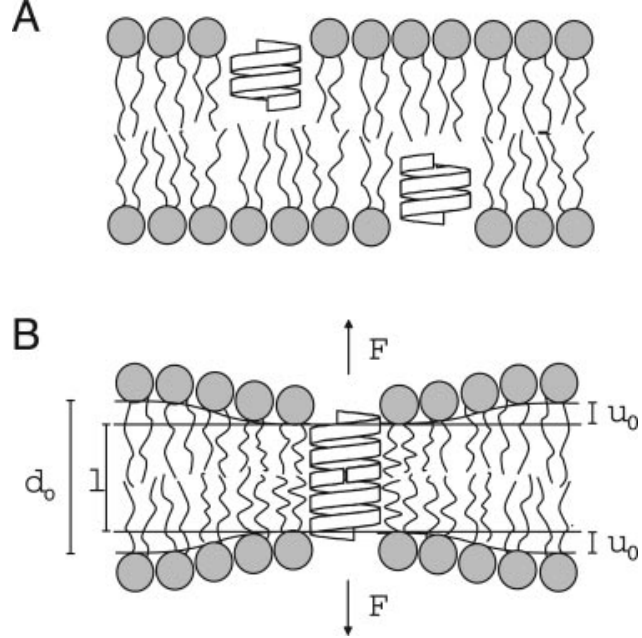

Fig. 1. Gramicidin channels as molecular force transducers. A, gramicidin monomers in a lipid bilayer. B, the bilayer imposes a disjoining force on the bilayer-spanning channel dimer. $\mathrm{d}_{0}$, bilayer hydrophobic thickness; 1 , channel hydrophobic length; $\mathrm{u}_{0}$, bilayer deformation; $\mathrm{F}$, disjoining force. [Modified from Lundbæk JA, Birn P, Hansen AJ, Søgaard R, Nielsen C, Girshman J, Bruno M, Tape S, Egebjerg J, Greathouse D, et al. (2004) Regulation of sodium channel function by bilayer elasticity: the importance of hydrophobic coupling. Effects of micelle-forming amphiphiles and cholesterol. J Gen Physiol 123:599-621. Copyright (C 2005 by the Rockefeller University Press. Used with permission.] is decreased (provided other factors affecting the dimer $\leftrightarrow$ monomer kinetics remain constant.)

Voltage-dependent sodium channels (VDSC) from rat skeletal muscle are regulated by amphiphiles that alter lipid bilayer stiffness (Lundbæk et al., 2004). A number of amphiphiles that decrease bilayer stiffness, such as Triton X-100, promote VDSC inactivation. The hyperpolarizing shift in the voltage dependence of inactivation correlates quantitatively with the decrease in bilayer stiffness. Cholesterol, which increases bilayer stiffness, has the opposite effect.

At the concentration at which many membrane proteins are affected, capsaicin and capsazepine decrease lipid bilayer stiffness measured using gA channels and promote VDSC inactivation, producing use-dependent current inhibition. For capsaicin, the quantitative relation between the decrease in bilayer stiffness and hyperpolarizing shift in the voltage dependence of inactivation conforms to that found for other amphiphiles (Lundbæk et al., 2004). We conclude that capsaicin can regulate VDSC function by altering lipid bilayer elasticity. The widespread regulation of membrane protein function by capsaicin and capsazepine may similarly be due to changes in bilayer elasticity.

\section{Materials and Methods}

Gramicidin Channel Experiments. $\left[\mathrm{Val}^{1}\right]$ gramicidin A ( $\left[\mathrm{Val}^{1}\right] \mathrm{gA}$ ) was purified as described by Greathouse et al. (1999). The enantiomeric analog $\left[\mathrm{D}-\mathrm{Val}^{1}\right] \mathrm{gA}{ }^{-}$, as well as the sequence-modulated analog $\left[\mathrm{Ala}^{1}\right] \mathrm{gA}$ and the sequence-shortened analog $\left[\mathrm{des}-\mathrm{Val}^{1}\right.$ $\left.\mathrm{Gly}^{2}\right] \mathrm{gA}^{-}$, were synthesized as described previously (Greathouse et al., 1999). gA channels in diphytanoylphosphatidylcholine (DPhPC)/ $n$-decane or dioleoylphosphatidylcholine (DOPC) $/ n$-decane bilayers were studied at $25 \pm 1^{\circ} \mathrm{C}$ (except for a few experiments at room temperature, $21-25^{\circ} \mathrm{C}$ ) using the punch-bilayer method. The total volume of lipid/decane solution was less than $2 \mu \mathrm{l}$ (or 1/1000-fold less than the volume of the aqueous solution.) The applied potential was $200 \mathrm{mV}$. The electrolyte solution was $1 \mathrm{M} \mathrm{NaCl}$, buffered to $\mathrm{pH} 7.0$ using $5 \mathrm{mM}$ HEPES (Sigma). Capsaicin or capsazepine were added to the chamber from a $10 \mathrm{mM}$ stock solution in ethanol. The ethanol concentration in the bilayer chamber never exceeded $1 \%(\mathrm{v} / \mathrm{v})$, a concentration that does not affect gA channel function. The current signal was filtered at 100 to $500 \mathrm{~Hz}$ and digitized at eight times the filter frequency. Single channel current transition amplitudes and lifetimes were determined online. The average channel lifetime $(\tau)$ was obtained by fitting a single exponential distribution to the life-

TABLE 1

Examples of structurally unrelated ion channels regulated by capsaicin and capsazepine

To avoid possible effects of TRPV1 receptors; the list is incomplete in that we focus on results obtained in cells in which the TRPV1 receptor has not been described. Furthermore, the examples chosen satisfy one or more of the following criteria: a) no activation of current by capsaicin or no change in resting membrane potential; b) no description of activation of current or change in resting membrane potential by capsaicin; c) effects of capsazepine similar to capsaicin; d) nonmammalian.

\begin{tabular}{|c|c|c|c|c|c|}
\hline Channel Type & Cell Type, Species & Capsaicin & Capsazepine & Criteria & Reference \\
\hline \multicolumn{6}{|c|}{$\mu M$} \\
\hline Delayed rectifier $\mathrm{K}^{+}$current & NG108-15 & 20 & 20 & $\mathrm{~b}, \mathrm{c}$ & Lo et al. (2003) \\
\hline$I_{\mathrm{K}(\mathrm{erg})}$ current & NG108-15 & 20 & & $\mathrm{~b}$ & Lo et al. (2003) \\
\hline L-type $\mathrm{Ca}^{2+}$ current & NG108-15 & 5 & 5 & $\mathrm{~b}, \mathrm{c}$ & Lo et al. (2003) \\
\hline Slow/fast $\mathrm{K}^{+}$current & Neuron, Xenopus laevis & $1-200$ & 50 & $\mathrm{~b}, \mathrm{c}, \mathrm{d}$ & Kuenzi and Dale (1996) \\
\hline $\mathrm{Ca}^{2+}$ current & Neuron, Xenopus laevis & $1-200$ & 50 & $\mathrm{~b}, \mathrm{c}, \mathrm{d}$ & Kuenzi and Dale (1996) \\
\hline Trans. outward $\mathrm{K}^{+}$current & Myocyte, rat & $1-30$ & & a & Wu et al. (1996) \\
\hline Late outward $\mathrm{K}^{+}$current & Myocyte, rat & $3-100$ & & $\mathrm{a}$ & Wu et al. (1996) \\
\hline Acetylcholine/adenosine, $\mathrm{K}^{+}$current & Myocyte, rat & $10-300$ & & $\mathrm{a}$ & Wu et al. (1996) \\
\hline Voltage-dependent $\mathrm{Na}^{+}$channel & Myocyte, rat & $0.4-4$ & & $\mathrm{~b}$ & Milesi et al. (2001) \\
\hline $\operatorname{Kv}(1.1,1.2,1.3,1.5,3.1)$ & L929, B82, MEL & $20-200$ & & $\mathrm{~b}$ & Grissmer et al. (1994) \\
\hline Nicotinic acetylcholine receptor & Pheochromocyte, rat & $1-30$ & & $\mathrm{~b}$ & Nakazawa et al. (1994) \\
\hline CFTR & NIH3T3, CHO & $25-200$ & & a & Ai et al. (2004) \\
\hline $\mathrm{I}_{\mathrm{CRAC}}$ & Jurkat T-cell, human & $2-200$ & $1-25$ & $\mathrm{a}, \mathrm{c}$ & Fischer et al. (2001) \\
\hline
\end{tabular}

CFTR, cystic fibrosis transmembrane conductance regulator. 
time histograms: $N(t) / N(0)=\exp (-t / \tau)$, where $N(t)$ is the number of channels with a lifetime longer than $t$.

The capacitance of $\mathrm{DPhPC} / n$-decane bilayers was measured by applying a sawtooth potential wave across bilayers with an area of $\sim 1.5 \mathrm{~mm}^{2}$ (Lundbæk and Andersen, 1994). The bilayer area was calculated from the two major axes measured using a microscope with a calibrated reticule.

Small Angle X-Ray Scattering. Samples for X-ray diffraction were prepared by mixing stock solutions of DOPE and DOPC (1:1) in $\mathrm{CHCl}_{3}$ with capsaicin or Triton $\mathrm{X}-100$, in ethanol, in a narrow glass tube (diameter, $2.5 \mathrm{~mm}$ ) to yield a total (lipid + capsaicin + Triton $\mathrm{X}-100)$ mass of $3 \mathrm{mg}$. After mixing at $60^{\circ} \mathrm{C}$, the solvent was removed by lyophilization; the powder resolubilized in cyclohexane at 100 $\mathrm{mg} / \mathrm{ml}$ and $0.6 \mathrm{mg}$ of tetradecane (20\% by weight of the amphipath) was added to induce the inverse hexagonal phase. Cyclohexane was removed by lyophilization at $-10^{\circ} \mathrm{C}$ to yield a white powder. Each sample was hydrated with $15 \mu \mathrm{l}$ of buffer $(20 \mathrm{mM}$ MOPS and $1 \mathrm{mM}$ EDTA, $\mathrm{pH}$ 7.0) and homogenized in five freeze/thaw/mix cycles. The mixture was transferred to 1-mm glass X-ray capillaries; after addition of $5 \mu \mathrm{l}$ of excess buffer, the capillary was sealed with vacuum grease backed by an epoxy plug. Three to five independent samples were prepared for each composition.

X-ray scattering data were obtained as described by Turner and Gruner, 1992). For each sample, the hexagonal lattice size, $d_{\text {hex }}$, was measured at $25.0 \pm 0.1^{\circ} \mathrm{C}$ after annealing at both 15 and $65^{\circ} \mathrm{C}$ (to confirm sample equilibration). For each composition, the uncertainty of $d_{\text {hex }}$ is reported as the standard deviation of the measurements on all samples. The radius, $R_{\mathrm{w}}$, and curvature, $C_{\mathrm{w}}$, of the lipid-water interface was computed as (Keller et al., 1993):

$$
R_{\mathrm{w}}=\frac{1}{C_{\mathrm{w}}}=-\left(\frac{d_{\mathrm{hex}}}{\sqrt{3}}-16 \AA\right)
$$

Expression of Sodium Channels. cDNA encoding the rat muscle sodium channel $\mu 1 \alpha$-subunit was a generous gift from G. Mandel (University of Stony Brook, Stony Brook, NY). Sodium channels were expressed in HEK293 cells, as described previously (Lundbæk et al., 2004). HEK293 cells were grown on cover slips in Dulbecco's modified Eagle's Medium supplemented with $10 \%$ fetal calf serum (Invitrogen, Carlsbad, CA), $100 \mathrm{U} / \mathrm{ml}$ penicillin (Invitrogen), and 100 $\mu \mathrm{g} / \mathrm{ml}$ streptomycin (Invitrogen) at $37^{\circ} \mathrm{C}$ ambient atmosphere with $5 \% \mathrm{CO}_{2}$. Stable clones expressing the sodium channel gene were

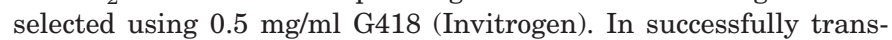
fected cells, used for the present experiments, the tetrodotoxinblocked peak currents were $\sim 100$ times larger than the peak currents in nontransfected cells.

Whole-Cell Voltage Clamp. Sodium channel currents were studied at room temperature $\left(21-25^{\circ} \mathrm{C}\right)$ using whole-cell voltage clamp. Voltage pulse generation, data acquisition, corrections for pipette and membrane capacitances and linear leakage currents, using a P-P4 protocol, were performed using an Axopatch 200A amplifier and pClamp 6.0 (Axon Instruments, Foster City, CA). Currents were filtered at $10 \mathrm{kHz}$ and sampled at $40 \mathrm{kHz}$. The tip resistance of the patch pipettes was 2 to $4 \mathrm{M} \Omega$. Only experiments with a series resistance below $4 \mathrm{M} \Omega$ (80\% compensation) and a voltage drop across the series resistance of less than $5 \mathrm{mV}$, after compensation, were used in the analysis. The electrode solution contained: $140 \mathrm{mM} \mathrm{CsCl}, 20 \mathrm{mM}$ HEPES, $11 \mathrm{mM}$ EGTA, $1 \mathrm{mM}$ $\mathrm{CaCl}_{2}, 1.8 \mathrm{mM}$ MgATP, $0.46 \mathrm{mM}, \mathrm{Na}_{3} \mathrm{GTP}, \mathrm{pH}$ 7.3. The bath solution contained $140 \mathrm{mM} \mathrm{NaCl}, 4 \mathrm{mM} \mathrm{KCl}, 2 \mathrm{mM} \mathrm{CaCl}, 2 \mathrm{mM} \mathrm{MgCl}_{2}, 10$ $\mathrm{mM}$ HEPES, and $10 \mathrm{mM}$ glucose, $\mathrm{pH}$ 7.4. Control or test solutions were applied to the cells using a fast superfusion system. Test solutions of capsaicin or capsazepine were prepared from a stock solution in ethanol. The ethanol concentration never exceeded $0.1 \%(\mathrm{v} / \mathrm{v})$ in the test solution, which did not affect sodium channel activation or inactivation (results not shown). The membrane leak conductance, measured by a 10 -ms hyperpolarizing pulse from the holding potential of -80 to $-90 \mathrm{mV}$, was not different in the control situation; after a 5 - to 10 -min application of $30 \mu \mathrm{M}$ capsaicin $(0.6 \pm 0.3$ vs. $0.4 \pm 0.2$ $\mathrm{nS}$ in the same six cells, $p>0.05$ ).

The voltage dependences of channel activation and steady-state (fast) inactivation were obtained as described by Lundbæk et al. (2004). In brief, the steady-state inactivation was obtained using a two-state Boltzmann expression:

$$
\frac{I(V)}{I(-130)}=\frac{A_{1}-A_{2}}{1+\exp \left\{\left(V-V_{\text {in }}\right) / S_{\text {in }}\right\}}+A_{2},
$$

where $I(V)$ and $I(-130)$ are the peak currents at prepulses of $V$ and $-130 \mathrm{mV}$, respectively, $A_{1}$ is the maximum value of $I(V) / I(-130)$ in the fit, $A_{2}$ is a constant offset because of noninactivating currents and current noise, $V_{\text {in }}$ is the voltage of half-maximal inactivation, and $S_{\text {in }}$ is a slope factor. The voltage dependence of activation was obtained using the conventional Hodgkin-Huxley relation:

$$
I(V)=\frac{G_{\max } \times\left(V-V_{\text {rev }}\right)}{1+\exp \left\{\left(V-V_{\text {act }}\right) / S_{\text {act }}\right\}}+A,
$$

where $I(V)$ is the peak current at the test potential $V, G_{\max }$ is the maximal conductance, $V_{\text {act }}$ is the voltage of half-maximal activation, $S_{\text {act }}$ is a slope factor, $E_{\text {rev }}$ is the reversal potential, and $A$ is a constant that includes a contribution from the peak-to-peak current noise.

Data Analysis. Curve fitting was done using the nonlinear leastsquares algorithm in Origin 7.0 (OriginLab Corp, Northampton, MA). Statistical analysis, using SigmaStat 2.0 (SPSS Inc., Chicago, IL), involved either Students $t$ test or one-way analysis of variance with Dunnetts's method as post hoc test. Unless otherwise noted, results are given as "mean \pm S.E.M., $(n)$ ", where $n$ is the number of cells.

\section{Results}

Capsaicin Modulation of gA Channel Function. Capsaicin increases $\left[\mathrm{Val}^{1}\right] \mathrm{gA}$ channel lifetime and activity (the time-averaged number of conducting channels) in DPhPC/ndecane bilayers (Fig. 2). Figure 2A shows current traces before and approximately 2 min after addition of $30 \mu \mathrm{M}$ capsaicin to both sides of a lipid bilayer. As seen in the current amplitude histograms (Fig. 2B) and lifetime distributions (Fig. 2C), both before and after capsaicin addition, only one predominant channel type is observed. Capsaicin does not affect the single channel conductance (Fig. 2B), showing that the interfacial dipole potential is not greatly altered (Hwang et al., 2003). The increase in channel lifetime is concentration-dependent (Fig. 2D): 10, 30, and $100 \mu \mathrm{M}$ capsaicin increase the lifetime to $125 \%, 200 \%$, and $600 \%$ of the control value, respectively.

Capsaicin (10-20 $\mu \mathrm{M})$ has been reported to form ion channels with a wide variety of conductances in lipid bilayers from a mixture of zwitterionic phospholipids (Feigin et al., 1995). Such channels were not observed in the present study. In the absence of gA, capsaicin-addition did not lead to discernible channel activity (and no new peaks were observed in the current transition amplitude histograms; Fig. 2B).

To determine whether the increase in $\left[\mathrm{Val}^{1}\right] \mathrm{gA}$ channel lifetime resulted from specific capsaicin-gA channel interactions, we measured the effects on channels formed by the enantiomeric $\left[\mathrm{D}-\mathrm{Val}^{1}\right] \mathrm{gA}{ }^{-}$. $\left[\mathrm{Val}^{1}\right] \mathrm{gA}$ and $\left[\mathrm{D}-\mathrm{Val}^{1}\right] \mathrm{gA}{ }^{-}$form right- and left-handed $\beta^{6.3}$-helical channels, respectively, and specific interactions would be expected to cause different effects on the two channel types. Capsaicin increases $\left[\mathrm{D}-\mathrm{Val}^{1}\right] \mathrm{gA}{ }^{-}$channel lifetime with a concentration dependence similar to that observed for $\left[\mathrm{Val}^{1}\right] \mathrm{gA}$ channels (Fig. 2D). To further investigate whether specific interactions 
could be involved, we studied the effects of substituting $\mathrm{Ala}^{1}$ for $\mathrm{Val}^{1}$. This change in side chains at the monomer-monomer interface causes a 4-fold decrease in channel lifetime, but the capsaicin-induced increase in [ $\left.\mathrm{Ala}^{1}\right] \mathrm{gA}$ channel lifetime is similar to that for $\left[\mathrm{Val}^{1}\right] \mathrm{gA}$ and $\left[\mathrm{D}-\mathrm{Val}^{1}\right] \mathrm{gA}{ }^{-}$channels (Fig. 2D). Thus, the effects of capsaicin do not depend on specific capsaicin-gA interactions. To determine whether the lipid acyl chains could be important, we also examined the changes in $\left[\mathrm{Val}^{1}\right] \mathrm{gA}$ lifetime in $\mathrm{DOPC} / n$-decane bilayers. These changes were similar to those observed in DPhPC bilayers (Fig. 2D). The effects of capsaicin do not depend on specific capsaicin-DPhPC interactions.

As depicted in Fig. 1, a gramicidin dimer both compresses and bends each bilayer leaflet; in reaction, the membrane pulls out on each half of the bilayer-spanning dimer with a disjoining force $(F)$. In this simple spring model, the channel appearance rate and lifetime are increased when the elastic disjoining force is reduced. The force's energetic contribution to channel dissociation can be approximated as by $F \times \delta$, where $\delta$ is the distance the two subunits must separate to reach the transition state for dimer dissociation, $\sim 1.6 \AA$ (Durkin et al., 1993). In the limiting case, when the lipid monolayer spontaneous curvature $\left(c_{0}\right)$ is zero, the relation between $F$, bilayer hydrophobic thickness $\left(d_{0}\right)$, and bilayer stiffness can be described using a simple spring model as:

$$
F=2 \times H_{\mathrm{B}} \times\left(d_{0}-l\right)
$$

where $l$ is the channel length and $H_{\mathrm{B}}$ is a phenomenological spring constant that, for a given inclusion and bilayer thickness, can be described in terms of the bilayer elastic moduli (Nielsen et al., 1998; Lundbæk and Andersen, 1999). For a given gramicidin channel, the channel length is effectively invariant (Katsaras et al., 1992) meaning that changes in $F$ could result from changes in $d_{0}$ and/or $H_{\mathrm{B}}$. (Eq. 2 describes a limiting case. Capsaicin produces changes in $c_{0}$ that will be described below. For the moment, we do not need to consider this aspect of capsaicin's effects).

To examine whether the capsaicin-induced increase in gA channel appearance rate and lifetime were associated with a decrease in $d_{0}$, we estimated the changes in bilayer hydrophobic thickness from specific capacitance $\left(C_{\mathrm{m}}\right)$ measurements on $\mathrm{DPhPC} / n$-decane bilayers (Lundbæk and Andersen, 1994), using the relation $C_{\mathrm{m}}=\epsilon_{\mathrm{o}} \times \epsilon_{\mathrm{r}} / d_{\mathrm{o}}$, where $\epsilon_{\mathrm{o}}$ and $\epsilon_{\mathrm{r}}$ denote the permittivity of free space and the relative dielectric constant of the bilayer hydrophobic core $(\approx 2.0)$. In the absence or presence of $40 \mu \mathrm{M}$ capsaicin, the bilayer specific capacitance was $4.2 \pm 0.1 \mathrm{nF} / \mathrm{mm}^{2}(n=5)$ or $4.1 \pm 0.1$ $\mathrm{nF} / \mathrm{mm}^{2}(n=5)$, respectively. Based on the invariance of $C_{\mathrm{m}}$, we conclude that capsaicin at concentrations up $40 \mu \mathrm{M}$ does not change the bilayer hydrophobic thickness. The increase in gA channel lifetime is probably due to decreased bilayer stiffness.

According to eq. 2, the capsaicin-induced changes in gA channel appearance rate and lifetime should vary as a function of the bilayer deformation $\left(d_{0}-l\right)$ associated with channel formation. A decrease in $H_{\mathrm{B}}$ would be predicted to alter channel function as follows. First, because the bilayer deformation involved in adjusting the bilayer hydrophobic thickness to the channel hydrophobic length $\left(d_{0}-l\right)$ is much larger than $\delta$ (Durkin et al., 1993), capsaicin's effect on channel appearance rates should be larger than on channel life- times. Second, because $\left(d_{0}-l\right)$ is larger for a short channel than for a long channel, capsaicin should have larger effects on the lifetime and appearance rate of the short channel. Third, capsaicin should have a larger effect on the ratio between the appearance rates of a short channel and a long channel than on the ratio between the corresponding lifetimes. These predictions hold.

To quantify the capsaicin-induced increase in $\left[\mathrm{Val}^{1}\right] \mathrm{gA}$ channel appearance rate, the average channel appearance rate in a DPhPC bilayers was determined over 14 min (Fig. 3). Then, $30 \mu \mathrm{M}$ capsaicin was added to both sides of the bilayer, the electrolyte solutions were stirred for $1 \mathrm{~min}$, and, beginning $6 \mathrm{~min}$ after the addition, the average channel appearance rate was measured again. Figure 3 shows the time course of the appearance rate, normalized by the average control value. Capsaicin caused a $5.5 \pm 0.5$-fold (mean \pm S.D., $n=3$ ) increase in channel appearance rate, which is greater than the 2-fold increase in lifetime (Fig. 2D).

The relation between the capsaicin-induced increase in gA channel lifetime and channel length was determined by comparing the effects on channels formed in the same DPhPC bilayer by subunits having either 15 amino acids ([Ala $\left.{ }^{1}\right] \mathrm{gA}$ ) or 13 amino acids ([des-Val $\left.\left.{ }^{1}-\mathrm{Gly}^{2}\right] \mathrm{gA}{ }^{-}\right)$. These two gramicidin analogs form channels of opposite helix sense that differ in length by $3.2 \AA$. (The opposite helix sense avoids formation of heterodimeric channels, which would complicate the data analysis.) Capsaicin increased the lifetimes of both $\left[\mathrm{Ala}^{1}\right] \mathrm{gA}$
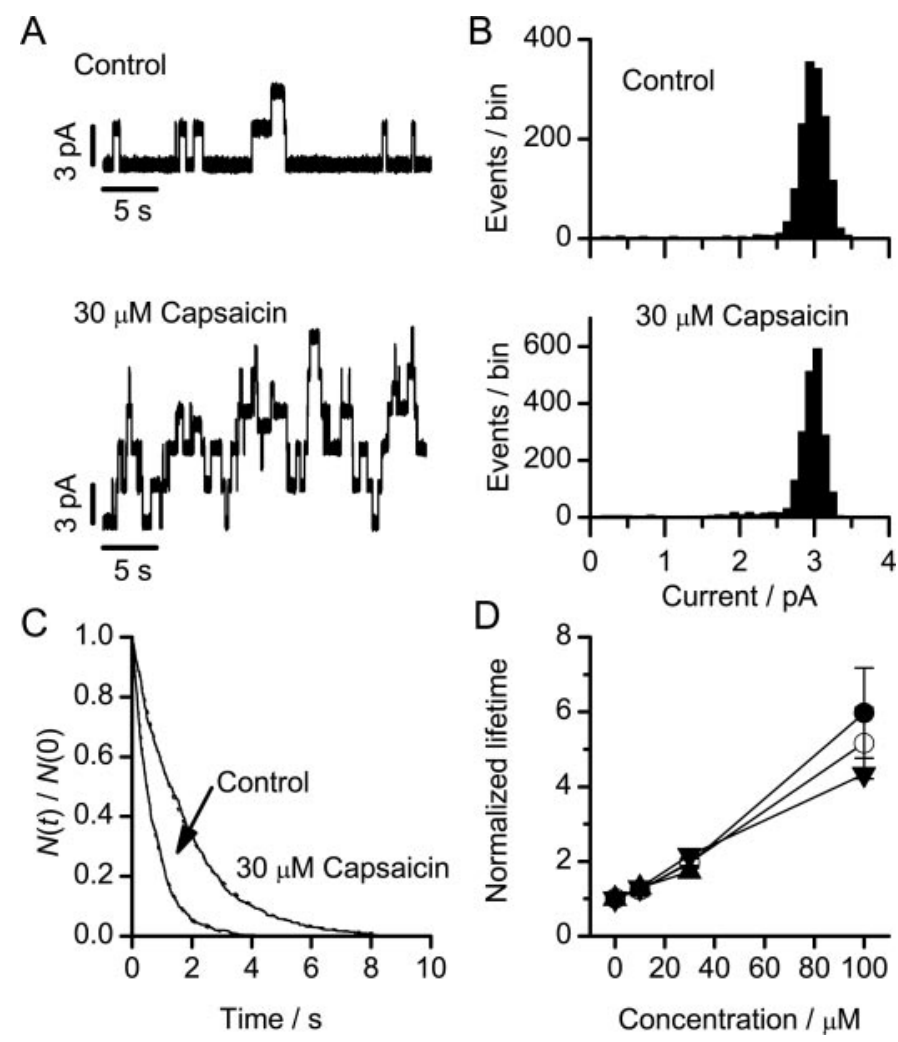

Fig. 2. Effect of capsaicin on gA channels in planar bilayers. A, singlechannel current traces showing the effect of capsaicin on gA channels in $\mathrm{DPhPC} / n$-decane bilayers. B, corresponding amplitude histograms. C, normalized lifetime distributions shown as survivor plots. The smooth curve is the fit of a single exponential distribution to the results. D, gramicidin channel lifetime as a function of capsaicin concentration. $\left[\mathrm{Val}^{1}\right] \mathrm{gA}$ in DPhPC; $\mathrm{O},\left[\mathrm{D}-\mathrm{Val}^{1}\right] \mathrm{gA}^{-}$in DPhPC; $\boldsymbol{\Delta},\left[\mathrm{Ala}^{1}\right] \mathrm{gA}$ in DPhPC; $\boldsymbol{\nabla}$ $\left[\mathrm{Val}^{1}\right] \mathrm{gA}$ in DOPC. 
and $\left[\right.$ des-Val $\left.{ }^{1}-\mathrm{Gly}^{2}\right] \mathrm{gA}{ }^{-}$channels ( $\tau_{15}$ and $\tau_{13}$, respectively), but the changes were larger for the shorter channel (Fig. 4A).

To compare the changes in the appearance rates of the two channel types, the appearance rates were determined based on $\sim 5$-min current traces recorded before and after capsaicin addition (the second recording was started $5 \mathrm{~min}$ after the addition). Figure $4 \mathrm{~B}$ shows the ratio of the normalized channel appearance rates for $\left[\right.$ des- $\left.\mathrm{Val}^{1}-\mathrm{Gly}^{2}\right] \mathrm{gA}^{-}$and $\left[\mathrm{Ala}^{1}\right] \mathrm{gA}$ channels $\left(f_{13} / f_{15}\right)$, evaluated from the appearance rates for the two channel types in the same bilayer. As would be expected for a mechanism based on changes in bilayer elasticity, the increase in appearance rate was larger for the

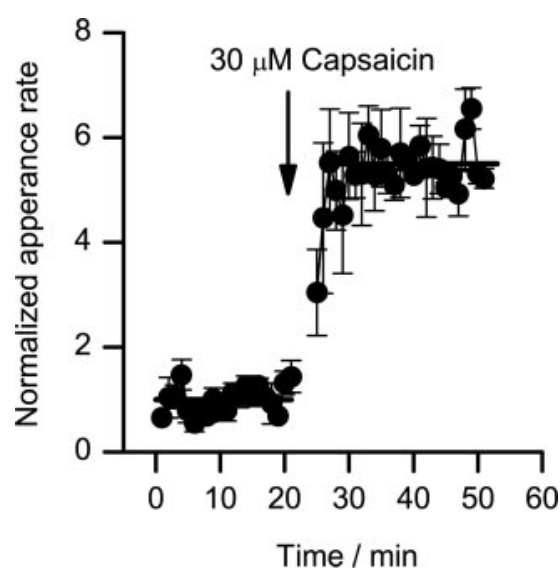

Fig. 3. Capsaicin modulation of $\left[\mathrm{Val}^{1}\right] \mathrm{gA}$ channel appearance rate. 100 $\mathrm{mV}$ applied potential.
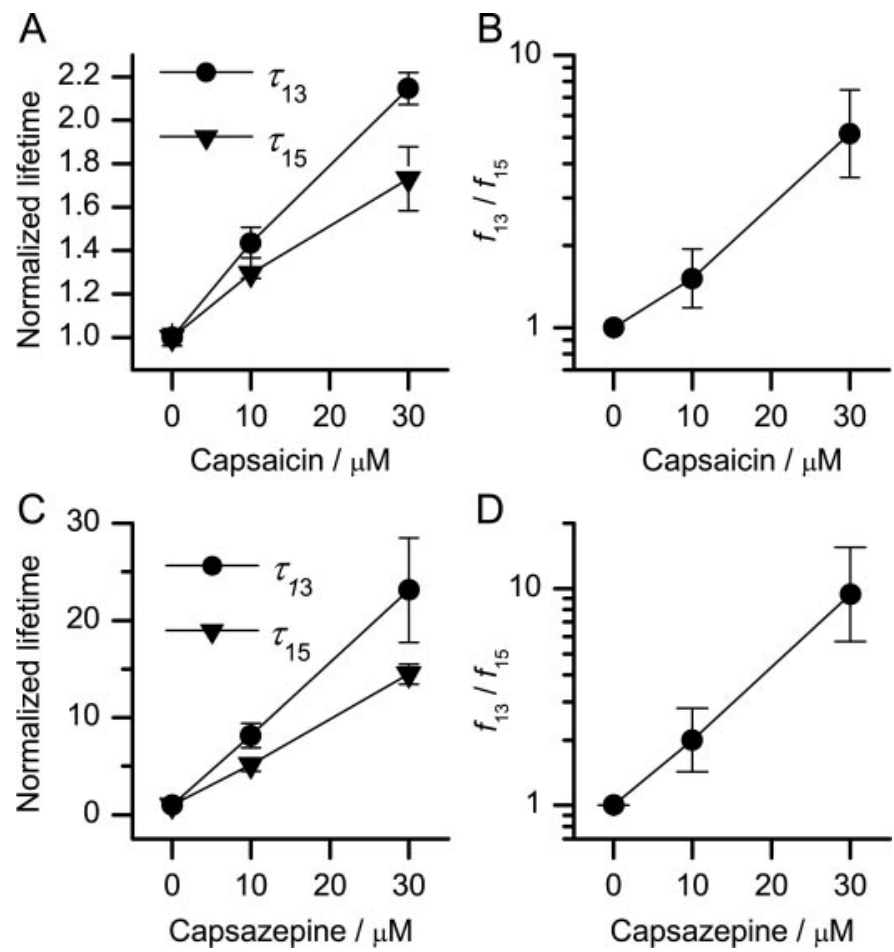

Fig. 4. Effects of capsaicin or capsazepine on channels formed by 15 amino acid monomers ([ $\left.\mathrm{Ala}^{1}\right] \mathrm{gA}$ (15 amino acids) and 13 amino acids $\left[\right.$ des- $\left.\mathrm{Val}^{1}-\mathrm{Gly}^{2}\right] \mathrm{gA}^{-}$. A, capsaicin-induced changes in channel lifetimes ( $\tau_{15}$ and $\tau_{13}$, respectively). B, capsaicin-induced changes in the ratio of appearance rates of $\left[\mathrm{des}-\mathrm{Val}^{1}-\mathrm{Gly}^{2}\right] \mathrm{gA}^{-}$and $\left[\mathrm{Ala}^{1}\right] \mathrm{gA}$ and channels $\left(f_{13} / f_{15}\right)$. C, capsazepine-induced changes in $\tau_{15}$ and $\tau_{13}$. D, capsazepineinduced changes in $\left(f_{13} / f_{15}\right)$. shorter channel; that is, $f_{13} / f_{15}$ is increased. Finally, the increase in $f_{13} / f_{15}$ (Fig. 4B) was larger than the increase in the corresponding lifetime ratio, $\tau_{13} / \tau_{15}$ (Fig. 4A).

Capsazepine Modulation of gA Channel Function. Capsazepine inhibits the activation of TRPV1 receptors $(\mathrm{Ca}-$ terina et al., 1997) but it emulates the effects of capsaicin on many other channels, suggesting that the bilayer elasticity is similarly altered (Table 1). Capsazepine would thus be expected to increase the appearance rates and lifetimes of both $\left[\mathrm{Ala}^{1}\right] \mathrm{gA}$ and $\left[\mathrm{des}-\mathrm{Val}^{1}-\mathrm{Gly}^{2}\right] \mathrm{gA}{ }^{-}$channels. This is the case.

In parallel to capsaicin, the capsazepine-induced increases in gA channel appearance rates and lifetimes (Fig. 4, C and $\mathrm{D})$, in DPhPC bilayers, were larger for the shorter channel: both $f_{13} / f_{15}$ and $\tau_{13} / \tau_{15}$ were increased-and the increase in $f_{13} / f_{15}$ was larger than the increase in $\tau_{13} / \tau_{15}$. We conclude that capsazepine, like capsaicin, decreases bilayer stiffness.

Changes in Monolayer Curvature. Bilayer-dependent changes in membrane protein function have often been associated with changes in spontaneous monolayer curvature or the average molecular shape of the bilayer-forming molecules (Gruner, 1985; Gruner, 1991; Lundbæk and Andersen, 1994; Bezrukov, 2000). Micelle-forming amphiphiles, such as Triton X-100, which would be expected to promote a positive monolayer curvature, increase gA channel appearance rate and lifetime (Lundbæk et al., 1996; Lundbæk et al., 2004). Capsaicin, in contrast, increases the propensity of phospholipids to form nonlamellar, inverted hexagonal $\left(H_{\mathrm{II}}\right)$ phases (Aranda et al., 1995), suggesting that a negative curvature is promoted. Thus, if the effects of capsaicin or Triton X-100 on gA channel function were due primarily to changes in spontaneous curvature, one would expect capsaicin and Triton $\mathrm{X}-100$ to have opposite effects on channel appearance rate and lifetime-contrary to what is observed. To explore this question further, we determined whether capsaicin and Triton $\mathrm{X}-100$ cause the expected changes in the spontaneous curvature of $H_{\mathrm{II}}$-phases formed by DOPC:DOPE/ $n$-tetradecane mixtures, using small angle X-ray diffraction (Figs. 5, A and $\mathrm{B})$.

The hexagonal lattice size $\left(d_{\text {hex }}\right)$ of DOPC:DOPE/ $n$-tetradecane (1:1) mixtures with varying mole-fractions of capsaicin or Triton X-100, was determined from the scattering intensity. Figure 5A shows results from three experiments: control conditions and in the presence of either capsaicin or Triton X-100. The radius of the lipid-electrolyte interface $\left(R_{\mathrm{W}}\right)$ and the lipid spontaneous curvature $\left(=1 / R_{\mathrm{W}}\right)$ were calculated from $d_{\mathrm{hex}}$, using eq. 1 (see Materials and Methods). As shown in Fig. 5B, the spontaneous curvature varies as a function of increasing mole-fractions of capsaicin or Triton $\mathrm{X}-100$ in the lipid phase. Capsaicin causes a negative change in curvature; Triton X-100 has the opposite effect. We conclude that the changes in gA channel function are not uniquely determined by changes in spontaneous curvature.

Capsaicin Alters Bilayer Stiffness. In summary, the capsaicin-induced modulation of gA channel lifetime does not involve specific capsaicin-channel interactions or changes in bilayer hydrophobic thickness. The relative effects of capsaicin and capsazepine on channel appearance and disappearance rates correlate with magnitude of the bilayer deformation involved-as would be expected for a mechanism involving changes in bilayer stiffness. Moreover, the rankorder of the capsaicin and capsazepine-induced changes in channel appearance rates and lifetimes and ratios of appear- 
ance rate to lifetime follow the general rules embodied in eq. 2 . We conclude that capsaicin and capsazepine, at the concentrations at which they exert their actions on many different ion channels, alter bilayer stiffness.

Capsaicin Modulation of Sodium Channel Function. The effects of capsaicin and capsazepine on rat muscle VDSC, expressed in HEK293 cells, were studied using whole-cell voltage clamp. TRPV1 receptors have not been described in this cell type and capsaicin did not alter the membrane background conductance (see Materials and Methods). Figure 6A shows current traces from an experiment in which 30 $\mu \mathrm{M}$ capsaicin was applied for $25 \mathrm{~s}$. Every $5 \mathrm{~s}$, the sodium channels were stimulated using a double-pulse protocol, where a 10 -ms test pulse to $+20 \mathrm{mV}$ was preceded by a $300-\mathrm{ms}$ prepulse to either -130 or $-60 \mathrm{mV}$ (the holding potential was $-80 \mathrm{mV}$ in all experiments). Figure $6 \mathrm{~B}$ shows the average effects on the normalized peak currents. A 25-s application of $30 \mu \mathrm{M}$ capsaicin caused a $12 \%$ or $37 \%$ decrease in the peak currents after the -130 or $-60 \mathrm{mV}$ prepulse, respectively. The prepulse-dependence of the inhibition shows that an inactivated channel state is promoted.

Capsaicin's modulation of VDSC inactivation was further investigated by determining the channels' steady-state availability in experiments in which a $300-\mathrm{ms}$ prepulse to potentials varying between -130 and $0 \mathrm{mV}$ was followed by a 10-ms test pulse to $+20 \mathrm{mV}$ (Fig. 7). In both the absence and presence of capsaicin, the voltage dependence of inactivation could be described by a two-state Boltzmann distribution (see

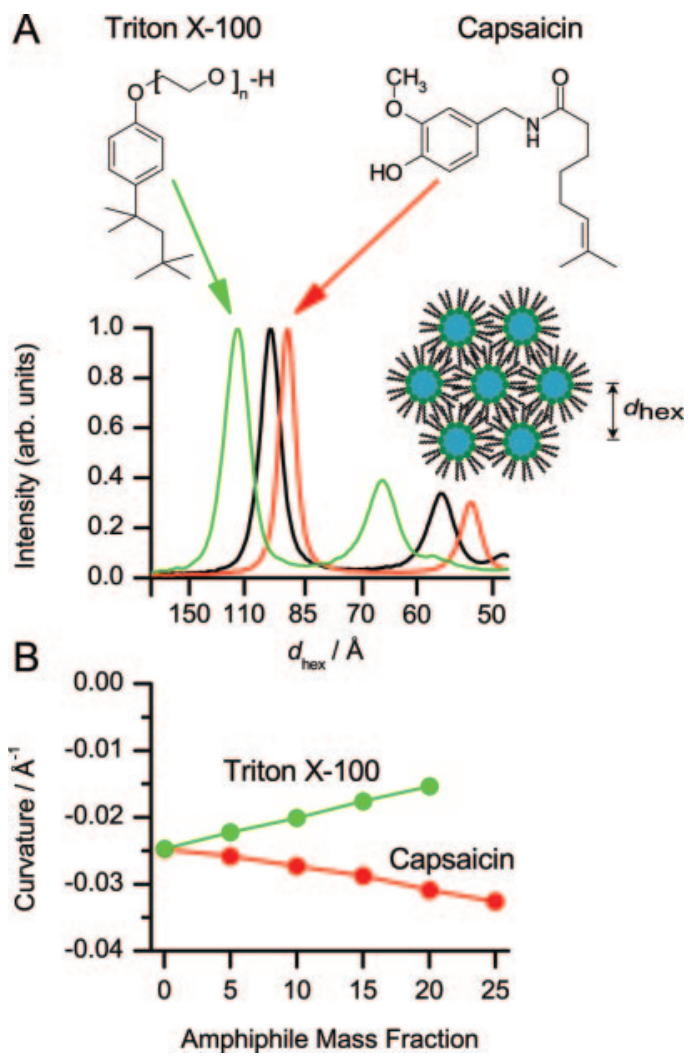

Fig. 5. Determination of spontaneous curvature changes. A, small-angle $\mathrm{X}$-ray scattering intensity; the inset illustrates the inverse hexagonal $\left(H_{\mathrm{II}}\right)$ phase being examined and the molecular structure of capsaicin and Triton X-100. B, spontaneous lipid curvature of DOPC-DOPE/ $n$-tetradecane $H_{\text {II }}$ phases as a function of the concentration of capsaicin or Triton $\mathrm{X}-100$ in the lipid phase.
Materials and Methods). Figure 7, A and B, shows the absolute and normalized peak current for a single experiment. In control cells, the voltage of half-maximal inactivation $\left(V_{\text {in }}\right)$ and the slope of the curve $\left(S_{\text {in }}\right)$ equaled $-59.6 \pm 2.3$ and $6.4 \pm$ $1.1 \mathrm{mV}(n=3)$, respectively. Capsaicin induced a hyperpolarizing shift in $V_{\text {in }}$-that is, inactivation is promoted. The concentration-dependent shift in $V_{\text {in }}$ is shown in Fig. $7 \mathrm{C}$ and is compared with the shift in timed control cells $V_{\text {in }} . S_{\text {in }}$ was not altered by 30 or $100 \mu \mathrm{M}$ capsaicin. Likewise, $30 \mu \mathrm{M}$ capsazepine caused a $-6.6 \pm 0.6-\mathrm{mV}$ shift in $V_{\text {in }}$ without altering $S_{\text {in }}(n=7)$.

The capsaicin-induced promotion of sodium channel inactivation was fully reversible after short exposures to capsaicin (Fig. 6) but only partially so after longer exposures (Fig. 7C). In the experiments shown in Fig. 7, capsaicin was applied for 5 to $15 \mathrm{~min}$ followed by 5 -min washout. We did not investigate longer washout times. The lack of total reversibility with longer applications may be due to capsaicin's hydrophobicity, which could slow the release from cellular membranes.

The channels' voltage activation was also investigated. In both the absence and presence of capsaicin, the peak-current voltage relations were well described by a conventional Hodgkin-Huxley type relation (see Materials and Methods) (Fig. 8). In the control situation, the voltage dependence of half-maximal activation $\left(V_{\text {act }}\right)$, slope of the peak current voltage relation $\left(S_{\text {act }}\right)$, and reversal potential $\left(E_{\text {rev }}\right)$ were $-16.0 \pm$ 3.0, $6.4 \pm 0.8$, and $84.7 \pm 2.9 \mathrm{mV}$, respectively $(n=3)$. Thirty micromolar capsaicin or capsazepine did not affect any of these values (Table 2 ), but $100 \mu \mathrm{M}$ capsaicin caused a $+4.0 \pm$ 1.0-mV change in $V_{\text {act }}$. In timed control experiments, none of these parameters were significantly altered (Table 2).

The Shift in Inactivation in Relation to the Changes in Bilayer Stiffness. The concentration dependence of the
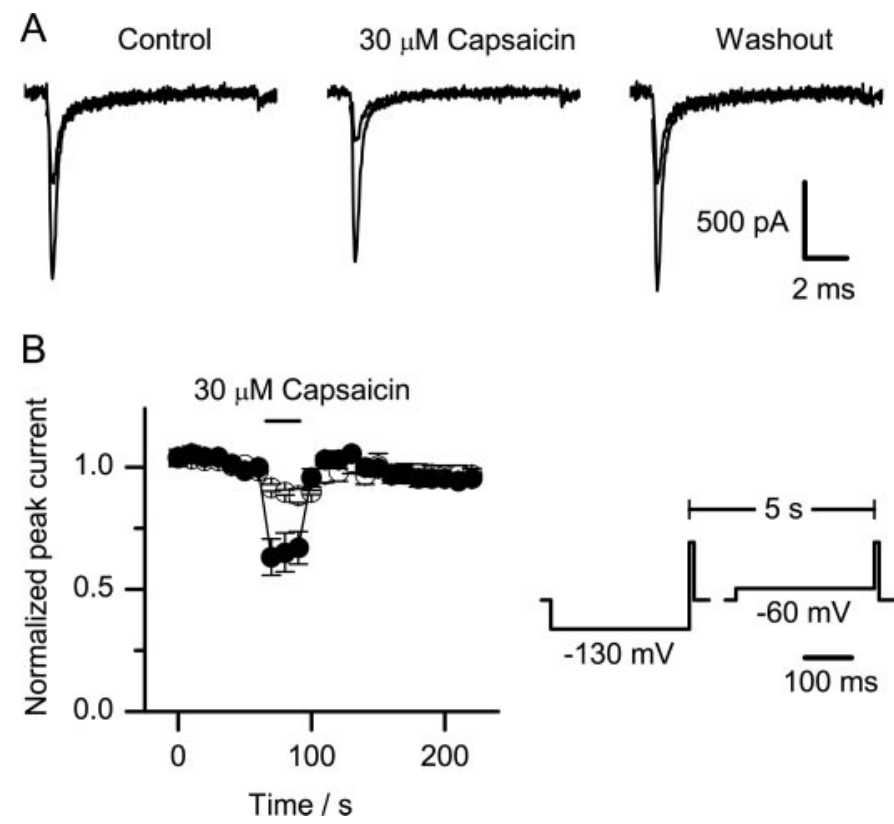

Fig. 6. Effect of capsaicin on VDSC currents. A, current traces showing the effects of $30 \mu \mathrm{M}$ capsaicin on rat muscle sodium channel currents in HEK293 cells. B, normalized peak currents. After a 300-ms prepulse to either -130 or $-60 \mathrm{mV}$, the channel availability was determined using a $+20-\mathrm{mV}$ test pulse. Capsaicin was applied for 25 s. (Mean \pm S.E.M., $n=4$.) 
shift in $V_{\text {in }}$ is different for capsaicin and Triton X-100 (Fig. 9A). Nevertheless, the relation between the capsaicin-induced changes in bilayer stiffness and in $V_{\text {in }}$ follow the relation previously found for Triton X-100 and other amphiphiles (Lundbæk et al., 2004). A change in the bilayer disjoining force, $F$, acting on a gA channel will lead to a change in channel lifetime, $\tau$, given by (Lundbæk and Andersen, 1999):
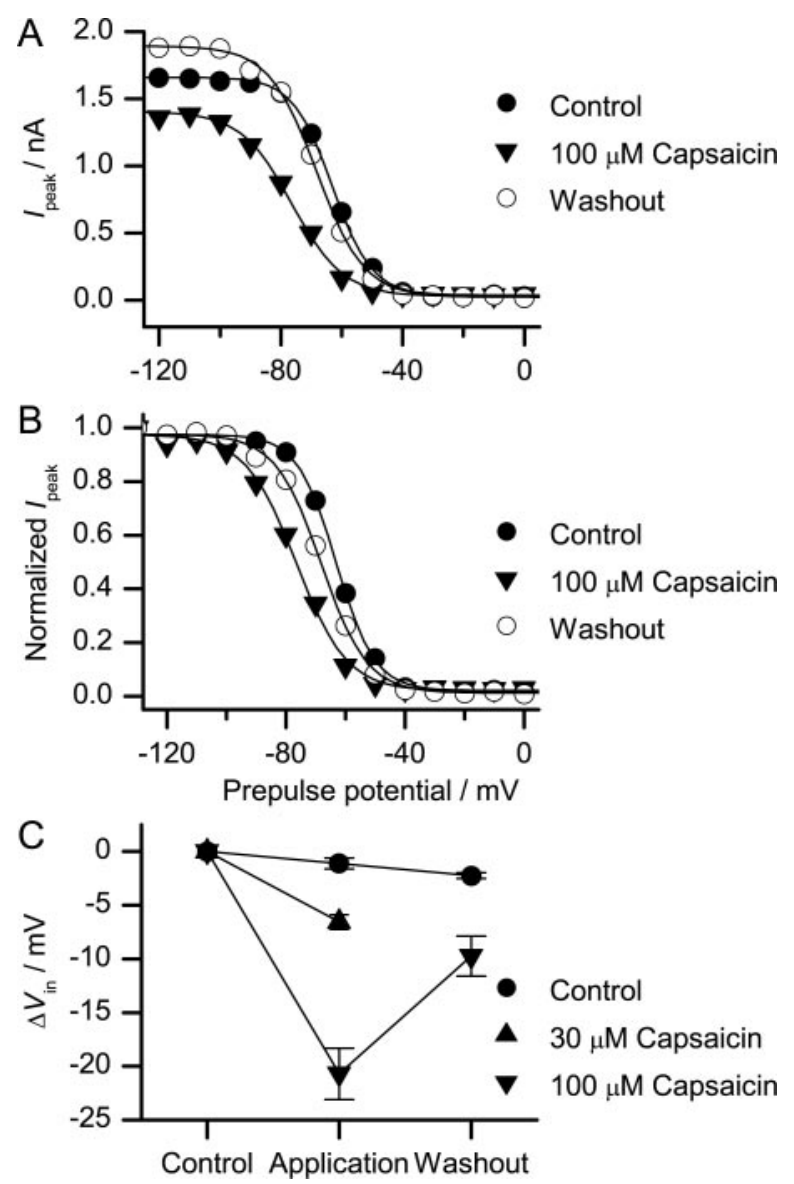

Fig. 7. Capsaicin modulation of sodium channel inactivation. A, peak currents versus prepulse potential, results from a single experiment. B, peak currents normalized to the current elicited after a $-130-\mathrm{mV}$ prepulse. C, concentration-dependent changes in the shift in $\Delta V_{\text {in }}$. Effects of capsaicin were compared with timed control experiments $(n=4$ and 2 $(100 \mu \mathrm{M})$ and 4 and $3(30 \mu \mathrm{M})$ during application and after washout, respectively. For control experiments, $n=5$ in all measurements.)

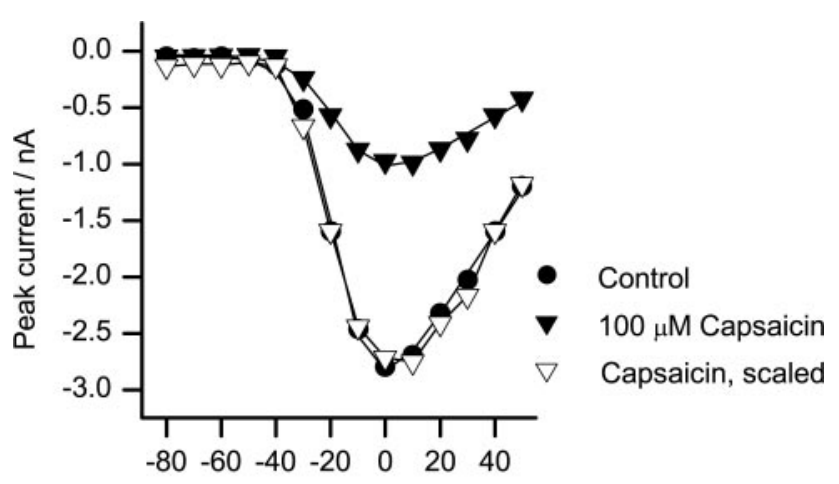

Potential / mV

Fig. 8. Effects of capsaicin on VDSC activation. Peak currents versus test potential. Results from a single experiment. The currents measured in the presence of $100 \mu \mathrm{M}$ capsaicin are scaled shown as measured $(\boldsymbol{\nabla})$ and scaled $(\nabla)$ to match the control peak inward current $(\bullet)$ recorded at $0 \mathrm{mV}$.

$$
\ln \left\{\frac{\tau_{\text {cap }}}{\tau_{\text {con }}}\right\} \approx-\frac{\left(F_{\text {cap }}-F_{\text {con }}\right) \times \delta}{R T},
$$

in which con and cap denote the absence and presence of capsaicin, $\delta$ is the distance the two subunits must separate to reach the transition state for dimer dissociation, and $R$ and $T$ are the gas constant and the absolute temperature, respectively. The right side of eq. 3 reflects the capsaicin-induced change in the bilayer contribution to the energetics of dimer dissociation, which at constant bilayer thickness is a measure of the capsaicin-induced change in bilayer elasticity. Figure 9B shows the capsaicin-induced shift in $V_{\text {in }}$ as a function of $\ln \left\{\tau_{\text {cap }} / \tau_{\text {con }}\right\}$ for gA channels in DOPC bilayers. The effect of $30 \mu \mathrm{M}$ capsaicin conforms to the quantitative relation previously shown for Triton X-100, reduced Triton X-100, $\beta$-octyl-glucoside, and Genapol X-100 (Lundbæk et al., 2004). As for the other compounds, the relation breaks down at higher capsaicin concentrations $(100 \mu \mathrm{M})$.

Time Course of the Return from Inactivation. As with to other amphiphiles that decrease bilayer stiffness (Lundbæk et al., 2004), the capsaicin-dependent promotion of VDSC inactivation is due to a slowed return from inactivation. This was shown in experiments in which the cells were depolarized every $5 \mathrm{~s}$ using double test pulses to $+10 \mathrm{mV}(10$ $\mathrm{ms}$ ) spaced by variable time periods at the holding potential of $-80 \mathrm{mV}(1.5-100 \mathrm{~ms})$. The time-dependent increase in channel availability was determined as the ratio of the first and the second peak current (Fig. 7). In the absence of capsaicin, the return from inactivation could be fitted using a double exponential time course $\left(I(t)=A_{1} \times \exp \left\{-t / \tau_{1}\right\}+(1-\right.$ $\left.\left.A_{1}\right) \times \exp \left\{-t / \tau_{2}\right\}\right)$, with time constants $\tau_{1}=4.4 \pm 0.1 \mathrm{~ms}$ and $\tau_{2}=80.4 \pm 10.9 \mathrm{~ms}$ and the amplitude $A_{1}=0.89 \pm 0.02$.

TABLE 2

Sodium channel activation in cells modified by capsaicin or capsazepine

Results are given as mean \pm S.E. of changes in individual cells.

\begin{tabular}{lccr}
\hline & $V_{\text {act }} / \mathrm{mV}$ & $S_{\text {act }} / \mathrm{mV}$ & \multicolumn{1}{c}{$E_{\text {rev }} / \mathrm{mV}$} \\
\hline Timed controls $(n=3)$ & $-1.5 \pm 0.8$ & $0.3 \pm 0.6$ & $3.0 \pm 3.4$ \\
$30 \mu \mathrm{M}$ Capsaicin $(n=4)$ & $-1.4 \pm 1.7$ & $0.8 \pm 0.7$ & $1.8 \pm 2.5$ \\
$100 \mu$ M Capsaicin $(n=4)$ & $4.0 \pm 1.0^{*}$ & $2.1 \pm 0.7$ & $-2.7 \pm 9.6$ \\
$30 \mu \mathrm{M}$ Capsazepine $(n=3)$ & $1.5 \pm 1.4$ & $0.0 \pm 0.2$ & $1.6 \pm 4.6$ \\
\hline
\end{tabular}

* Significantly different from the change in timed control cells; $P<0.05$.

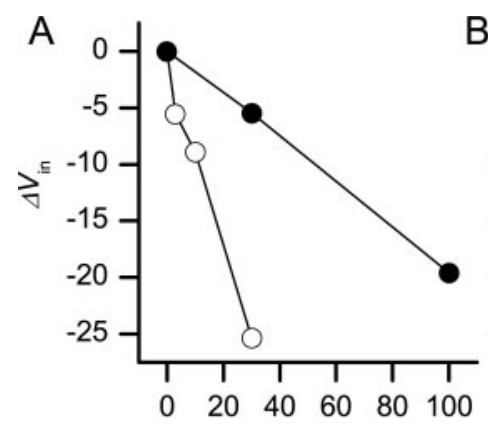

Concentration / $\mu \mathrm{M}$

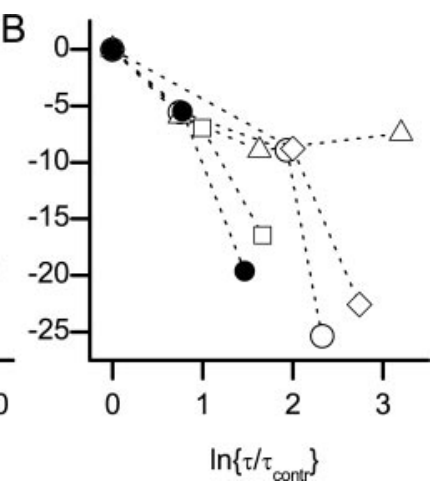

Fig. 9. A, shift in the voltage dependence of inactivation, $\Delta V_{\text {in }}$, versus concentration of capsaicin $(-)$ or Triton X-100 (○) (from Lundbæk et al., 2004). B, $\Delta V_{\text {in }}$ versus $\ln \left\{\tau / \tau_{\text {control }}\right\}$ in DOPC/n-decane bilayers. $\bullet$, capsaicin (gA results from Fig. 2D). Data for Triton X-100 (O), reduced Triton X-100 $(\diamond), \beta$-octyl glucoside $(\triangle)$ and Genapol X-100 $(\square)$ are from Lundbæk et al. (2004). 
Sixty micromolar capsaicin reversibly slowed the return from inactivation (Fig. 10). In the presence of capsaicin, $\tau_{1}=$ $12.4 \pm 0.6 \mathrm{~ms}, \tau_{2}=68.9 \pm 9.2 \mathrm{~ms}$, and $A_{1}=0.88 \pm 0.04$ (mean \pm range, $n=2$.)

Frequency-Dependent Current Inhibition. The capsaicin-induced promotion of sodium channel inactivation and decrease in the rate of return from the inactivated state suggest that the current inhibition might be frequency-dependent. Figure 11A shows the effects of $30 \mu \mathrm{M}$ capsaicin on the peak currents induced by 10 -ms test pulses to $+20 \mathrm{mV}$ using pulse frequencies of 10 to $66 \mathrm{~Hz}$. All results are normalized by the magnitude of the initial peak current in the control situation. Using $10 \mathrm{~Hz}$ stimulation, capsaicin reduced both the first peak and last peak current in a 10-s pulse train by $10 \%$. Using $66 \mathrm{~Hz}$ stimulation, the first peak current was decreased by $10 \%$ and the last peak current by $75 \%$. The capsaicin-induced current inhibition therefore depends on the stimulus frequency. Similar but more pronounced frequency-dependent current inhibition was observed with 30 $\mu \mathrm{M}$ capsazepine (Fig. 11B).

\section{Discussion}

At the concentrations at which they modulate the function of many unrelated proteins, capsaicin and capsazepine shift the gA monomer $\leftrightarrow$ dimer equilibrium in favor of the bilayerspanning dimers, meaning that bilayer stiffness is decreased. Stereospecific mechanisms can be excluded. The changes in gA channel function vary as a function of the hydrophobic mismatch between bilayer thickness and channel length. We conclude that capsaicin and capsazepine alter the bilayer elasticity sufficiently to change the conformational preference of an embedded protein. Capsaicin and capsazepine cause a hyperpolarizing shift in VDSC inactivation and a frequency-dependent inhibition of VDSC currents. For capsaicin, the relation between the shift in inactivation and the change in bilayer stiffness conforms to the results obtained with a variety of structurally unrelated compounds.

Bilayer Physical Properties and Membrane Protein Function. Capsaicin, capsazepine, and other amphiphiles, such as Triton X-100, will tend to localize at the bilayer/ solution interface. In the case of capsaicin, the polar vanillyl group is likely to reside at the interface with the acyl chain inserted into the bilayer core. Adsorption of capsaicin to lipid bilayers thus would be expected to affect bilayer physical

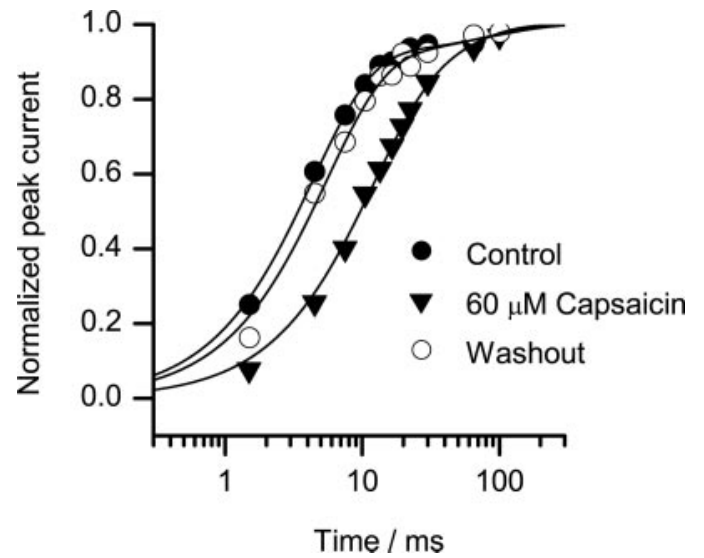

Fig. 10. Effect of $60 \mu \mathrm{M}$ capsaicin on the time course of return from inactivation. Results from a single experiment. properties-and capsaicin alters lipid bilayer "fluidity" (Meddings et al., 1991), phase preference (Aranda et al., 1995), and curvature (Fig. 5). That capsaicin alters bilayer physical properties provides a basis for the regulation of membrane protein function (Gruner, 1985, 1991; Andersen et al., 1992), although the causal relation between bilayer physics and protein function remains difficult to identify (Gruner, 1991). A change in bilayer fluidity, for example, might alter gA channel lifetimes, but it cannot alter the gA monomer $\leftrightarrow$ dimer equilibrium (Fig. 2A) or VDSC inactivation (Fig. 7) following Lee (1991). Capsaicin and Triton X-100 have opposite effects on lipid spontaneous curvature yet quantitatively similar effects on the relation between gA and VDSC function. Although gA channel function can be regulated by changes in spontaneous curvature (Lundbæk et al., 1997), the capsaicin-induced changes in channel function must be dominated by some other change in bilayer properties.

What Bilayer Properties Do gA Channels Monitor? When a gA channel forms, the bilayer deforms locally. This elastic deformation incurs an energetic cost, meaning that the bilayer imposes a disjoining force on the channel. For a given deformation, the magnitude of this force is determined by the bilayer continuum elastic properties (bilayer hydrophobic thickness, monolayer spontaneous curvature, and the compression and bending moduli), and by local changes in lipid packing (Huang, 1986; Nielsen et al., 1998; Nielsen and Andersen, 2000). These properties are determined by the profile of intermolecular interactions among the bilayer molecules-meaning that they cannot be varied independently. It thus becomes difficult to predict how an amphiphile will alter the disjoining force acting on a gA channel-or more generally the bilayer elastic response to membrane protein
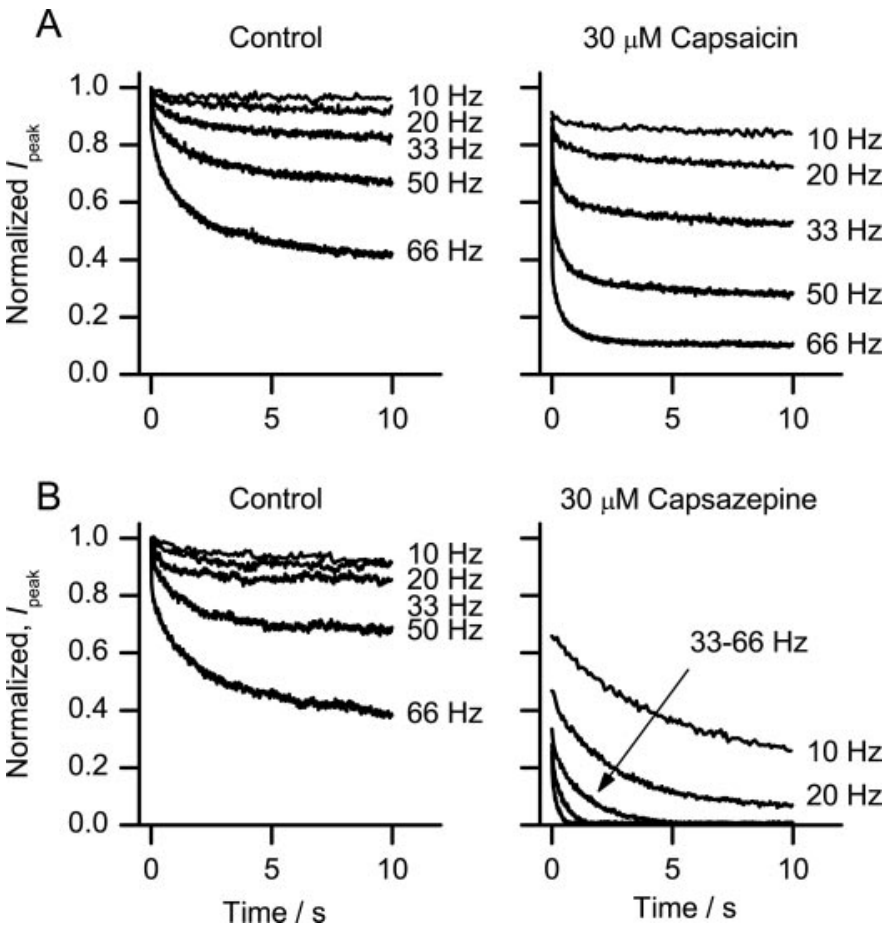

Fig. 11. Stimulus frequency dependence of capsaicin- and capsazepinedependent VDSC current inhibition. Normalized peak currents versus time, using stimulation frequencies varying between 10 and $66 \mathrm{~Hz}$. Effects of $30 \mu \mathrm{M}$ capsaicin (mean \pm S.E., $n=3)$ or capsazepine $(n=1)$ compared with the control situation in the same cells. 
conformational changes. However, because the disjoining force is directly reflected in the channel appearance rate and lifetime, gA channels become in situ force transducers to monitor net changes in bilayer elasticity.

It is noteworthy that a correlation between capsaicin's effects on gA channels and other membrane proteins (including VDSCs) does not imply similar "conformational" changes in the two channel types. Changes in bilayer elasticity, reported as altered stiffness measured using gA channels, reflect changes in the profile of the intermolecular interactions among the bilayer molecules, which determine the energetic cost of bilayer deformations caused also by more complex protein conformational changes.

The simplicity of the gA monomer $\leftrightarrow$ dimer transition allows for insights into the effects of amphiphiles on bilayer elasticity and imbedded proteins. When the spontaneous curvature of each monolayer of the membrane, $c_{0}$, is zero, the disjoining force on a bilayer-spanning channel may described by eq. 2 , but in general, $F$ will be given by Nielsen and Andersen (2000) and Lundbæk et al. (2004):

$$
F=2 \times H_{\mathrm{B}} \times\left(d_{0}-l\right)+H_{\mathrm{X}} \times c_{0},
$$

where $H_{\mathrm{X}}$ like $H_{\mathrm{B}}$ is a function of the bilayer elastic moduli (and $d_{0}$ and the channel radius). $F$ therefore depends on $c_{0}$. Furthermore, amphiphiles that reversibly adsorb at the bilayer/solution interface alter the bilayer elastic moduli, (Ly and Longo, 2004) in addition to their possible effects on $d_{0}$ and $c_{0}$. Changes in bilayer composition therefore alter $H_{\mathrm{B}}$, $H_{\mathrm{X}}, d_{0}$, and $c_{0}$, which makes it difficult, if not impossible, to predict a priori the relative importance of the two terms on the right side of eq. 4 . That Triton X-100 and capsaicin have opposite effects on $c_{0}$ and yet exert similar effects on gA channels and VDSC nevertheless allows us to conclude that the changes in channel function do not result from changes in $c_{0}$ per se but rather are dominated by changes in $H_{\mathrm{B}}$.

Frequency-Dependent Current Inhibition. Because capsaicin stabilizes the inactivated state of the VDSC and slows return from inactivation, one would expect capsaicin to produce use-dependent inhibition of VDSC currents. That is the case (Fig. 11). A drug-induced use-dependent current inhibition often is attributed to the drug's binding to a specific channel state (the open channel in the case of usedependent block, or the inactivated state, thereby stabilizing the nonconducting channel states). However, any manipulation that stabilizes the inactivated channel (and slows the return from inactivation) will produce a use-dependent current inhibition at sufficiently high stimulus frequencies. This allows for new perspectives in ion channel (and membrane protein) regulation, in that the bilayer becomes an allosteric regulator of membrane protein function.

In particular, use-dependent current inhibition does not mean that capsaicin binds to the channel [e.g., in the intracellular access path to the selectivity filter (Hille, B., 2001)], thereby causing open channel block. First, the prepulse dependence of the inhibition (Fig. 6) shows that the effects of capsaicin can be ascribed almost totally to promotion of inactivation and does not depend on channel opening. Second, the quantitative relation between the effects of capsaicin on inactivation and bilayer stiffness is very similar to that of structurally unrelated amphiphiles (Fig. 9), suggestive of a general, physical interaction, as opposed to a more specific chemical interaction (binding). Third, slowed return from inactivation (Fig. 10) in itself can account for the frequency dependence.

Inhibition of VDSC by local anesthetic has been described by the "modulated receptor hypothesis" (see also Hille, 2001) (Scheme 1), where current inhibition rises because the $I^{*}$

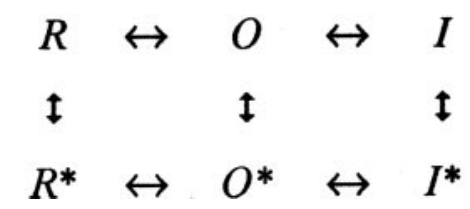

Scheme 1. $R, O$, and $I$ denote the resting, open, and inactivated states, respectively, and the asterisks denote drug-bound (or drug-modified) states.

state is stabilized (relative to $R^{*}$ and $O^{*}$ ) compared with the unmodified states. Pharmaceutically induced changes in the energetic cost of bilayer deformations associated with protein conformational changes can be described using a similar model (Scheme 2), in which the bilayer becomes an allosteric modulator of membrane protein function.

\begin{tabular}{lllll|}
\hline$R$ & $\leftrightarrow$ & $O$ & $\leftrightarrow$ & $I$ \\
& & & & \\
$R^{*}$ & $\leftrightarrow$ & $O^{*}$ & $\leftrightarrow$ & $I^{*}$ \\
\hline
\end{tabular}

Scheme 2. $R, O$, and $I$ denote the resting, open, and inactivated states, respectively, and the three asterisks denote the VDSC in a lipid bilayer of altered stiffness.

Implications for Membrane Proteins and Amphiphiles. At the concentrations often used in physiological studies, capsaicin alters bilayer elasticity sufficiently to alter the conformational preference of a bilayer-embedded protein and promotes VDSC inactivation in quantitative correlation with the changes in bilayer stiffness. In the present context, VDSC are generic representatives of membrane proteins and

TABLE 3

Structurally unrelated amphiphiles that decrease bilayer stiffness and promote VDSC inactivation

\begin{tabular}{|c|c|c|c|c|}
\hline \multirow{2}{*}{ Amphiphile } & \multicolumn{2}{|c|}{ gA } & \multicolumn{2}{|c|}{ VDSC } \\
\hline & $k_{\text {ass }}$ & $k_{\text {diss }}$ & Activation & Inactivation \\
\hline Capsaicin & $\uparrow$ & $\downarrow$ & $(0)$ & $\uparrow$ \\
\hline Capsazepine & $\uparrow$ & $\downarrow$ & 0 & $\uparrow$ \\
\hline Lysophosphatidylcholine & $\uparrow a$ & $\downarrow a$ & $\downarrow^{b}$ & $\uparrow b$ \\
\hline Diazepam & $\uparrow^{c}$ & $\downarrow^{c}$ & & $\uparrow^{d}$ \\
\hline Chlorpromazine & $\uparrow c$ & $\downarrow^{c}$ & $0^{e}$ & $\uparrow e$ \\
\hline Pentobarbital & $\uparrow^{c}$ & $\downarrow^{c}$ & $0^{f}$ & $\uparrow f$ \\
\hline Triton X-100 & $\uparrow g$ & $\downarrow^{g}$ & $0^{g}$ & $\uparrow^{g}$ \\
\hline Reduced Triton X-100 & $\uparrow g$ & $\downarrow g$ & $0^{g}$ & $\uparrow g$ \\
\hline$\beta$-Octyl-glucoside & $\uparrow g$ & $\downarrow g$ & $0^{g}$ & $\uparrow g$ \\
\hline Genapol X-100 & $\uparrow g$ & $\downarrow g$ & $0^{g}$ & $\uparrow g$ \\
\hline Genistein & $\uparrow h$ & $\downarrow^{d}$ & $0^{i}$ & $\uparrow^{i}$ \\
\hline Cholesterol-depletion & $\uparrow g_{3} j$ & $\downarrow g, j$ & $0^{g}$ & $\uparrow g$ \\
\hline Cholesterol & $\downarrow g, j$ & $\uparrow^{g} j$ & $\downarrow^{g}$ & $\downarrow g$ \\
\hline
\end{tabular}

${ }^{a}$ Lundbæk and Andersen (1994).

${ }^{b}$ Shander et al. (1996).

${ }^{c}$ Sun et al. (2002).

${ }^{d}$ Backus et al. (1991)

e Ogata and Narahashi (1989).

${ }^{f}$ Rehberg et al. (1995).

${ }^{g}$ Lundbæk et al. (2004)

${ }^{h}$ Hwang et al. (2003).

${ }^{i}$ Wang et al. (2003).

${ }^{j}$ Lundbæk et al. (1996). 
one would expect that 1) micromolar capsaicin should alter the function of many different membrane proteins and that 2) other amphiphiles, which increase gA channel lifetime, also promote VDSC inactivation and have widespread effects on other membrane proteins. As shown in Table 1, capsaicin indeed regulates many ion channels in systems where TRPV1-mediated regulation is unlikely (the authors can provide a substantially larger number of examples on request).

As shown in Table 3, amphiphiles that increase gA channel lifetime indeed promote VDSC inactivation. Activation of VDSC is in general not affected. (All these amphiphiles regulate a number of membrane proteins, and some interact with specific receptors at submicromolar concentrations; the examples listed describe effects of micromolar concentrations on VDSC and gA channels.) Cholesterol, which decreases gA channel lifetime, in contrast, inhibits VDSC inactivation. To the best of our knowledge, only one group of amphiphiles does not conform to this general relation: long-chain alcohols decrease gA channel lifetime but promote VDSC inactivation (Lundbæk et al., 2004). They also promote VDSC activation, indicating that other mechanisms are involved. Indeed, although changes in bilayer elasticity provide a general mechanism whereby amphiphiles can regulate membrane protein function, this is not the only mechanism, of course. Capsazepine, for example, alters bilayer elasticity; it also inhibits capsaicin-activation of TRPV1 receptors, presumably through more or less specific interactions. This combination of effects could make capsazepine useful for distinguishing between capsaicin's specific (TRPV1-mediated) and more nonspecific regulation of cell function.

\section{Conclusion}

We conclude that capsaicin and capsazepine can modulate membrane protein function by altering bilayer elasticity and that capsaicin can change VDSC function by this mechanism. Other amphiphiles that increase gA channel appearance rate and lifetime may act by a similar mechanism. These results add to the evidence suggesting that bilayer elasticity is a general regulator of membrane protein function.

\section{Acknowledgments}

We thank Drs. C. Nielsen for stimulating discussions and J. Egebjerg for assistance with the molecular biology.

\section{References}

Ai T, Bompadre SG, Wang X, Hu S, Li M, and Hwang TC (2004) Capsaicin potentiates wild-type and mutant cystic fibrosis transmembrane conductance regulator chloride-channel currents. Mol Pharmacol 65:1415-1426.

Andersen OS, Sawyer DB, and Koeppe RE 2nd (1992) Modulation of channel function by the host bilayer, in Biomembrane Structure and Function (Easwaran KRK and Gaber B eds), pp 227-244, Adenine Press, Schenectady, NY.

Aranda FJ, Villalain J, and Gomez-Fernandez JC (1995) Capsaicin affects the structure and phase organization of phospholipid membranes. Biochim Biophys Acta 1234:225-234.

Backus KH, Pflimlin P, and Trube G (1991) Action of diazepam on the voltagedependent $\mathrm{Na}^{+}$current. Comparison with the effects of phenytoin, carbamazepine, lidocaine and flumazenil. Brain Res 548:41-49.

Bezrukov SM (2000) Functional consequences of lipid packing stress. Curr Opin Coll Interface Sci 5:237-243.

Caterina MJ, Schumacher MA, Tominaga M, Rosen TA, Levine JD, and Julius D (1997) The capsaicin receptor: a heat-activated ion channel in the pain pathway. Nature (Lond) 389:816-824.

Durkin JT, Providence LL, Koeppe RE II, and Andersen OS (1993) Energetics of heterodimer formation among gramicidin analogues with an $\mathrm{NH}_{2}$-terminal addition or deletion. Consequences of a missing residue at the join in channel. $\mathrm{J} \mathrm{Mol}$ Biol 231:1102-1121.

Evans EA and Hochmuth RM (1978) Mechanochemical properties of membranes. Curr Top Membr Transp 10:1-64.
Feigin AM, Aronov EV, Bryant BP, Teeter JH, and Brand JG (1995) Capsaicin and its analogs induce ion channels in planar lipid bilayers. Neuroreport 6:2134-2136. Fischer BS, Qin D, Kim K, and McDonald TV (2001) Capsaicin inhibits Jurkat T-cell activation by blocking calcium entry current I(CRAC). J Pharmacol Exp Ther 299:238-246.

Greathouse DV, Koeppe IRE, Providence LL, Shobana S, and Andersen OS (1999) Design and characterization of gramicidin channels. Methods Enzymol 294:525550

Grissmer S, Nguyen AN, Aiyar J, Hanson DC, Mather RJ, Gutman GA, Karmilowicz MJ, Auperin DD, and Chandy KG (1994) Pharmacological characterization of five cloned voltage-gated $\mathrm{K}^{+}$channels, types $\mathrm{Kv} 1.1,1.2,1.3,1.5$ and 3.1 , stably expressed in mammalian cell lines. Mol Pharmacol 45:1227-1234.

Gruner SM (1985) Intrinsic curvature hypothesis for biomembrane lipid composition: a role for nonbilayer lipids. Proc Natl Acad Sci USA 82:3665-3669.

Gruner SM (1991) Lipid membrane curvature elasticity and protein function, in Biologically Inspired Physics (Peliti L ed), pp 127-135, Plenum Press, New York.

Hille B (2001) Ionic Channels of Excitable Membranes. Sinauer Associates, Inc., Sunderland, MA

Huang HW (1986) Deformation free energy of bilayer membrane and its effect on gramicidin channel lifetime. Biophys J 50:1061-1070.

Hwang TC, Koeppe RE, and Andersen OS (2003) Genistein can modulate channel function by a phosphorylation-independent mechanism: importance of hydrophobic mismatch and bilayer mechanics. Biochem 42:13646-13658.

Katsaras J, Prosser RS, Stinson RH, and Davis JH (1992) Constant helical pitch of the gramicidin channel in phospholipid bilayers. Biophys $J$ 61:827-830.

Keller SL, Bezrukov SM, Gruner SM, Tate MW, Vodyanoy I, and Parsegian VA (1993) Probability of alamethicin conductance states varies with nonlamellar tendency of bilayer phospholipids. Biophys $J$ 65:23-27.

Kuenzi FM, and Dale N (1996) Effect of capsaicin and analogues on potassium and calcium currents and vanilloid receptors in Xenopus embryo spinal neurones. $\mathrm{Br} J$ Pharmacol 119:81-90

Lee AG (1991) Lipids and their effects on membrane proteins: evidence against a role for fluidity. Prog Lipid Res 30:323-348.

Lee AG (2003) Lipid-protein interactions in biological membranes: a structural perspective. Biochim Biophys Acta 1612:1-40.

Lo YK, Chiang HT, and Wu SN (2003) Effect of arvanil ( $N$-arachidonoyl-vanillylamine), a nonpungent anandamide-capsaicin hybrid, on ion currents in NG108-15 neuronal cells. Biochem Pharmacol 65:581-591.

Lundbæk JA and Andersen OS (1994) Lysophospholipids modulate channel function by altering the mechanical properties of lipid bilayers. J Gen Physiol 104:645-673. Lundbæk JA, Birn P, Girshman J, Hansen AJ, and Andersen OS (1996) Membrane stiffness and channel function. Biochemistry 35:3825-3830.

Lundbæk JA, Maer AM, and Andersen OS (1997) Lipid bilayer electrostatic energy, curvature stress and assembly of gramicidin channels. Biochemistry 36:5695-5701.

Lundbæk JA and Andersen OS (1999) Spring constants for channel-induced lipid bilaye deformations. Estimates using gramicidin channels. Biophys $J$ 76:889-895.

Lundbæk JA, Birn P, Hansen AJ, Søgaard R, Nielsen C, Girshman J, Bruno M, Tape S, Egebjerg J, Greathouse D, et al. (2004) Regulation of sodium channel function by bilayer elasticity: the importance of hydrophobic coupling. Effects of micelleforming amphiphiles and cholesterol. J Gen Physiol 123:599-621.

Ly HV and Longo ML (2004) The Influence of short-chain alcohols on interfacial tension, mechanical properties, area/molecule and permeability of fluid lipid bilayers. Biophys J 87:1013-1033

Meddings JB, Hogaboam CM, Tran K, Reynolds JD, and Wallace JL (1991) Capsaicin effects on non-neuronal plasma membranes. Biochim Biophys Acta 1070:43-50.

Milesi V, Rebolledo A, Alvis AG, Raingo J, and Grassi de Gende AO (2001) Voltageactivated sodium current is inhibited by capsaicin in rat atrial myocytes. Biochem Biophys Res Commun 282:965-970.

Mouritsen O and Bloom M (1984) Mattress model of lipid-protein interactions in membranes. Biophys J 46:141-153.

Nakazawa K, Inoue K, Koizumi S, Ikeda M, and Inoue K (1994) Inhibitory effects of capsaicin on acetylcholine-evoked responses in rat phaeochromocytoma cells. $\mathrm{Br} J$ Pharmacol 113:296-302.

Nielsen C and Andersen OS (2000) Inclusion-induced bilayer deformations: effects of monolayer equilibrium curvature. Biophys J 79:2583-2604.

Nielsen C, Goulian M, and Andersen OS (1998) Energetics of inclusion-induced bilayer deformations. Biophys J 74:1966-1983.

Ogata N and Narahashi T (1989) Block of sodium channels by psychotropic drugs in single guinea-pig cardiac myocytes. Br J Pharmacol 97:905-913.

Rehberg B, Bennett E, Xiao YH, Levinson SR, and Duch DS (1995) Voltage- and frequency-dependent pentobarbital suppression of brain and muscle sodium channels expressed in a mammalian cell line. Mol Pharmacol 48:89-97.

Shander G, Undrovinas AI, and Makielski JC (1996) Rapid onset of lysophosphatidylcholine-induced modification of whole cell cardiac sodium current kinetics. $J$ Mol Cell Cardiol 28:743-753.

Sun Y, Jogini V and Andersen OS (2002) Pharmacological modification of gramicidin channel function (Abstract). Biophys J 82:550a.

Turner DC and Gruner SM (1992) X-ray diffraction reconstruction of the inverted hexagonal (HII) phase in lipid-water systems. Biochemistry 31:1340-1355.

Wang Y, Wagner MB, Kumar R, Cheng J, and Joyner RW (2003) Inhibition of fast sodium current in rabbit ventricular myocytes by protein tyrosine kinase inhibitors. Pflueg Arch Eur J Physiol 446:485-491.

Wu S, Chen I, Lo Y, and Yu H (1996) The characteristics in the inhibitory effects of capsaicin on voltage-dependent $\mathrm{K}^{+}$currents in rat atrial myocytes. Environ Toxicol Pharmacol 2:39-47.

Address correspondence to: Jens A. Lundbæk, Department of Physiology and Biophysics, Weill Medical College, Cornell University, 1300 York Avenue, New York, NY 10021. E-mail: lundbaek@dadlnet.dk 